Supporting Information for

\title{
Assessment of the Maximal Activity of Complex IV in the Inner Mitochondrial Membrane by Tandem Electrochemistry and Respirometry
}

\author{
Nathan L. Frantz, ${ }^{1}$ Gabrielle Brakoniecki, ${ }^{1}$ Dawei Chen, ${ }^{1}$ and Denis A. Proshlyakov ${ }^{2,1 *}$ \\ ${ }^{1}$ Department of Chemistry and ${ }^{2}$ Department of Physiology, Michigan State University, East Lansing, MI, \\ *Address correspondence to Denis A. Proshlyakov, Dept. of Physiology, Michigan State University, 567 \\ Wilson Rd., East Lansing, MI 48824, dapro@ chemistry.msu.edu
}

\section{Table of Contents}

Figure S1: Schematic of counter and reference electrodes

Table S1: Electrochemically active surface area of electrode materials

Figure S2: TMPD concentration dependence of autoxidation in the presence of Na ascorbate in airequilibrated sample

Figure S3: Autoxidation rates in microfluidic conditions

Figure S4: Correlation between oxygen consumption rates simultaneously determined from the transferred charge and fluorescence quenching....

Figure S5: Mass transfer schematics of mediated mitochondrial electrochemistry

Figure S6: Electrochemical response of artificial mediators

Figure S7: Electrochemical response of cytochrome $c$ on modified electrodes.

Figure S8: Correlation of mitochondrial oxygen consumption rates observed upon chemical and electrochemical reduction at various TMPD concentrations

Figure S8: Linearity of OCRs with mitochondrial concentration at various TMPD concentrations .....S-11

References 


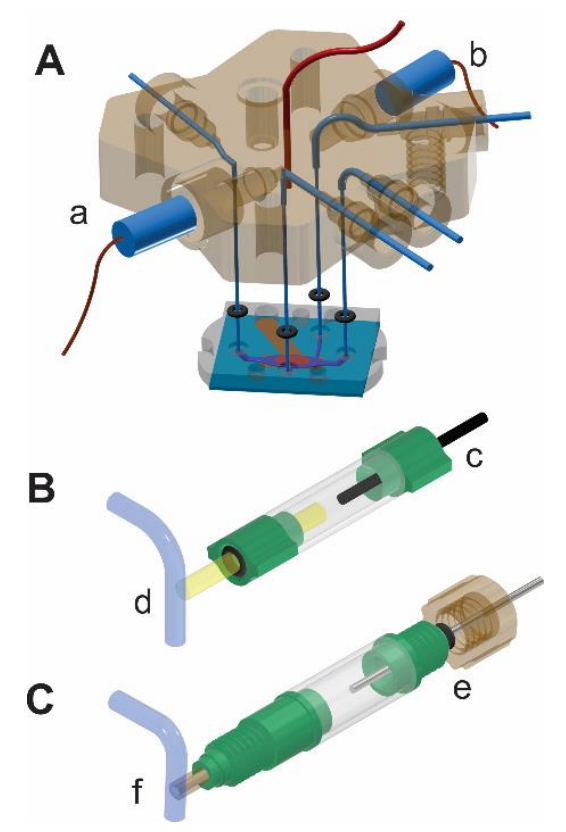

Figure S1: Schematics of counter and reference electrodes. A) Microfluidic manifold housing the counter electrode (a) and the reference electrode (b). B) Counter electrode consisting of pencil lead (c) and sol-gel plug (d). C) The $\mathrm{Ag} / \mathrm{AgCl}$ reference electrode consisting of oxidized $\mathrm{Ag}$ wire (e) and cased $\mathrm{Pt}$ wire (f).

Both counter and reference capillaries extended past the corresponding electrode assembly to the separate sealable ports, allowing air bubbles to be flushed from the capillaries with supporting electrolyte.

Counter electrode (Figure S1, B) A $1.3 \mathrm{~mm}$ diameter graphite pencil lead (c) was partially submerged in $0.5 \mathrm{M} \mathrm{KCl}$ in a sealed compartment and the dry end was connected to a potentiostat. A short section of tetramethyl-orthosilicate (TMOS) sol-gel cast in a $2.7 \mathrm{~mm}$ diameter polyethylene tubing created an ion-permeable partition (d) between the counter electrode and the capillary leading to the chip. The precursor stock solution was prepared by mixing $1.57 \mathrm{~mL}$ TMOS, $350 \mu \mathrm{L}$ MilliQ water, and $11 \mu \mathrm{L} 0.04 \mathrm{M} \mathrm{HCl}$, followed by sonication for $30 \mathrm{~min}$ in an ice bath in a fume hood. An equal volume of $0.5 \mathrm{M} \mathrm{KCl}$ in $150 \mathrm{mM}$ potassium phosphate buffer, $\mathrm{pH} \mathrm{7,} \mathrm{was} \mathrm{added} \mathrm{and} \mathrm{the} \mathrm{mixture} \mathrm{was} \mathrm{transferred} \mathrm{into} \mathrm{polyethylene} \mathrm{tubing} \mathrm{for}$ polymerization. The sol-gel-filled tubing was cut to size with a blade and stored in $0.5 \mathrm{M} \mathrm{KCl}$, $150 \mathrm{mM}$ potassium phosphate, $\mathrm{pH} \mathrm{7,} \mathrm{until} \mathrm{use.} \mathrm{Sol-gel} \mathrm{partition} \mathrm{in} \mathrm{the} \mathrm{manifold} \mathrm{was} \mathrm{always} \mathrm{kept}$ wet.

Reference electrode (Figure $\mathrm{S} 1, \mathrm{C}$ ) A saturated $\mathrm{KCl} \mathrm{Ag/AgCl}$ reference electrode was prepared by the oxidation of $\mathrm{Ag}$ wire (e) in $1 \mathrm{M} \mathrm{HCl}$. The reference electrode compartment was partitioned from microfluidic capillary by a Radel-cased Pt wire (f). A $0.25 \mathrm{~mm}$ Pt wire was inserted into a Radel tubing with an undersized I.D. (1/16 in. O.D.) upon softening by dry heat. After cooling, Pt core and Radel casing were cut to $2 \mathrm{~mm}$ lengths and assembled in the manifold by compressing 1/16" I.D. o-ring. Reference electrode was tested periodically by cyclic voltammetry (CV) of 1 $\mathrm{mM} \mathrm{Fe}(\mathrm{CN}) 6^{-3 /-4}$ in a conventional electrochemical cell over $\mathrm{GC}$ electrode and regenerated as necessary. 
Table S1: Electrochemically active surface area of electrode materials. $\mathrm{Ru}\left(\mathrm{NH}_{3}\right)_{6}{ }^{2+/ 3+}$ and $\mathrm{Fe}(\mathrm{CN}) 6^{-3 /-4}$ in $0.5 \mathrm{M} \mathrm{KCl}$ were used as the inner- and outer-sphere electron transfer probes ${ }^{1}$ to determine the electrochemically active surface area of Au electrodes modified with fumed silica (AuFS) or carbon ink before (raw $\mathrm{AuCi}$ ) and after (activated $\mathrm{AuCi}$ ) activation. Results for glassy carbon electrode are shown for comparison. All electrodes had a geometric surface area of $0.2 \mathrm{~cm}^{2}$. Cyclic voltammetry measurements were performed on $1 \mathrm{mM}$ analytes in a three-electrode bulk cell using carbon rod as a counter electrode and a saturated $\mathrm{KCl} \mathrm{Ag/AgCl} \mathrm{reference} \mathrm{electrode.} \mathrm{Surface} \mathrm{area} \mathrm{was} \mathrm{calculated} \mathrm{using} \mathrm{the} \mathrm{Randles-Sevcik}$ equation. AuCi was activated by the application of a $+2 \mathrm{~V}$ potential for 120 seconds in a $50 \mathrm{mM}$ potassium phosphate ( $\mathrm{pH} 7.0$ ) solution. Diffusion coefficients of $7.2 \times 10^{-6}$ and $9.1 \times 10^{-6} \mathrm{~cm}^{2} \mathrm{sec}^{-1}$ were used for $\mathrm{Fe}(\mathrm{CN})_{6}^{-3 /-4}$ and $\mathrm{Ru}\left(\mathrm{NH}_{3}\right)_{6}{ }^{2+/ 3+}$ respectively. ${ }^{2,3}$

\begin{tabular}{c|c|c} 
Effective area $\left(\mathrm{cm}^{2}\right)$ & $\mathrm{Ru}\left(\mathrm{NH}_{3}\right)_{6}^{2+/ 3+}$ & $\mathrm{Fe}(\mathrm{CN})^{-3 /-4}$ \\
\hline \hline glassy carbon & $0.197 \pm 0.001$ & $0.201 \pm 0.007$ \\
\hline AuFS & $0.140 \pm 0.006$ & $0.213 \pm 0.005$ \\
\hline raw AuCi & $0.134 \pm 0.010$ & $0.138 \pm 0.003$ \\
activated AuCi & $0.209 \pm 0.014$ & $0.228 \pm 0.005$
\end{tabular}




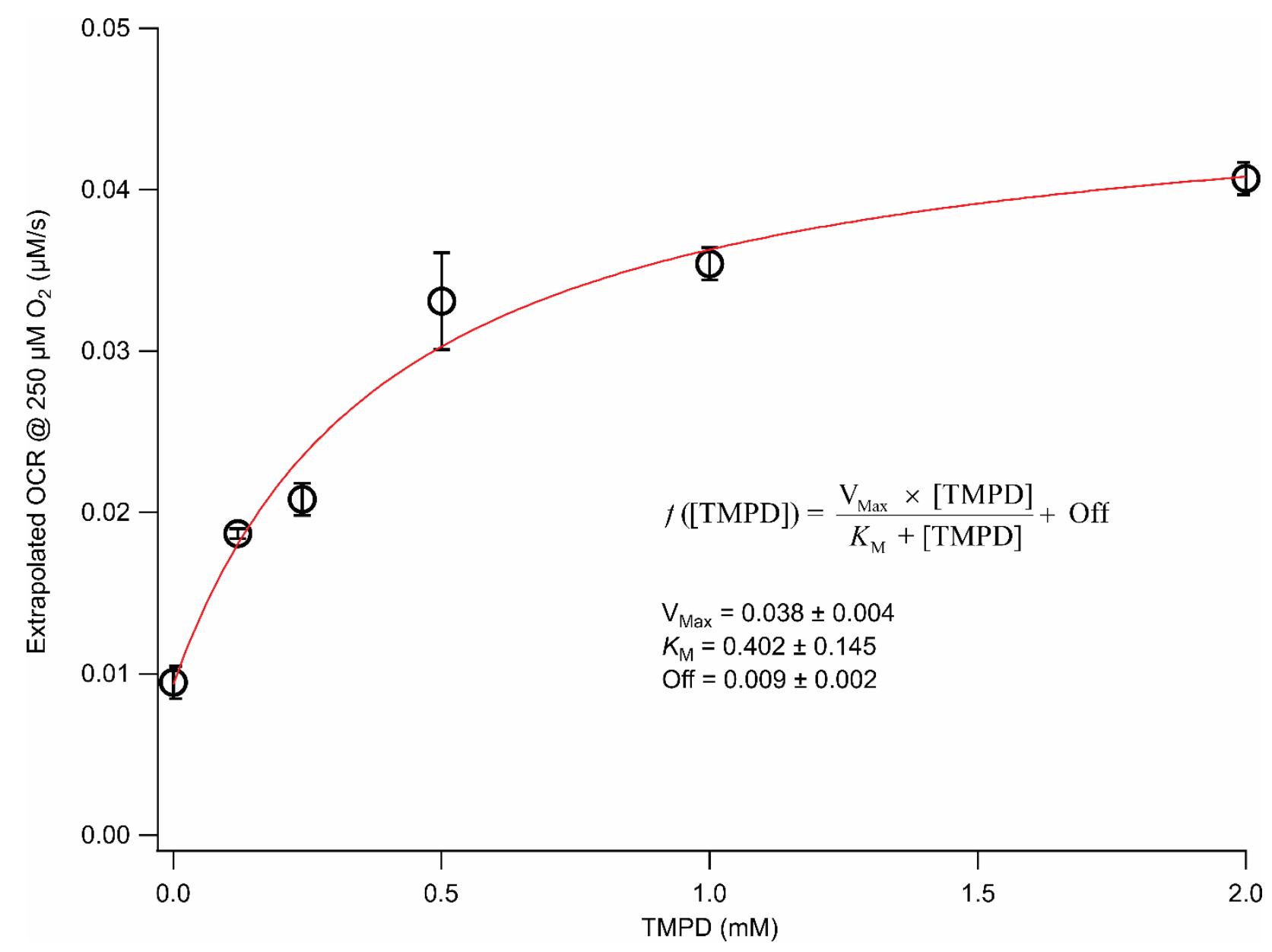

Figure S2: TMPD concentration dependence of autoxidation in the presence of $\mathrm{Na}$ ascorbate in airequilibrated sample. The OCR resulting autoxidation in the presence of $4 \mathrm{mM} \mathrm{Na}$ ascorbate, $25 \mu \mathrm{M}$ cytochrome c, and varying concentration of TMPD in the reaction buffer, $\mathrm{pH} 7.4$, were recorded using Clark electrode at room temperature. Since actual $\mathrm{O}_{2}$ concentration varied between individual measurements depending on the concentration of TMPD, measured OCR were extrapolated from the average $\mathrm{O}_{2}$ concentration during OCR measurement to the air equilibrated conditions $\left(250 \mu \mathrm{M} \mathrm{O}_{2}\right)$ using reported linear dependence of autoxidation on $\mathrm{O}_{2} \cdot{ }^{4}$ Measurements were conducted at $\mathrm{O}_{2}$ of $>170 \mu \mathrm{M}$ and non-linearity of autoxidation at low $\mathrm{O}_{2}$ concentrations $(<50 \mu \mathrm{M})$ was neglected. 


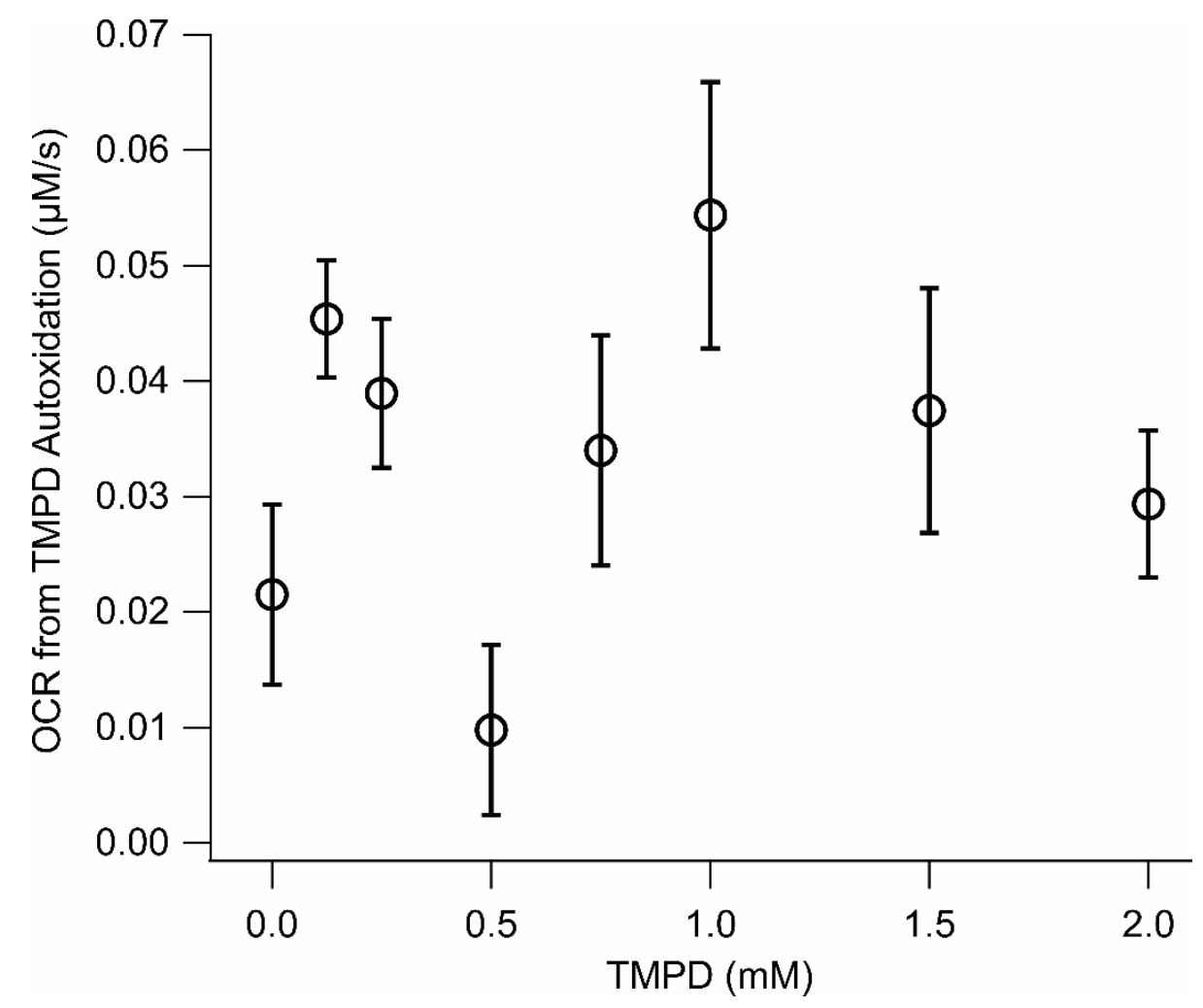

Figure S3: Autoxidation rates in microfluidic conditions. OCR from autoxidation in the presence of 25 $\mu \mathrm{M}$ cytochrome $c$ and varying TMPD concentrations were measured in the microfluidic chamber in the reaction buffer, $\mathrm{pH} 7.4$, under the applied potential of $-150 \mathrm{mV}$ vs $\mathrm{Ag} / \mathrm{AgCl}$ (saturated $\mathrm{KCl}$ ) reference electrode. Autoxidation OCR were measured immediately before corresponding measurements of mitochondrial samples. 


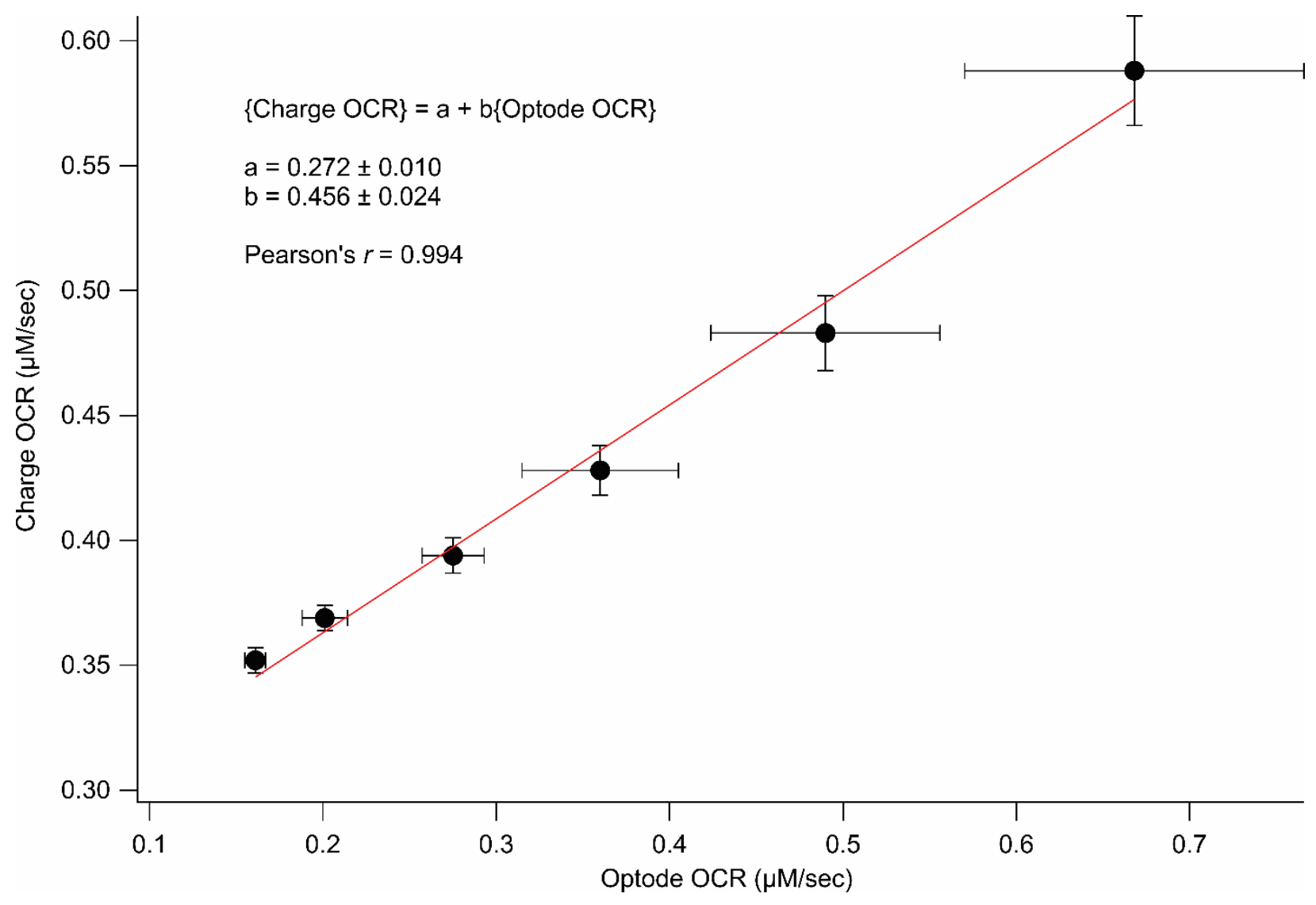

Figure S4: Correlation between oxygen consumption rates simultaneously determined from the transferred charge and fluorescence quenching. Plot shows relationship between charge OCR and optode OCR presented in Figure. 3C in the main text. Pearson's $r=0.994$ exceeds the critical value of 0.707 for this sample $(n=6, \alpha=0.5)$. 


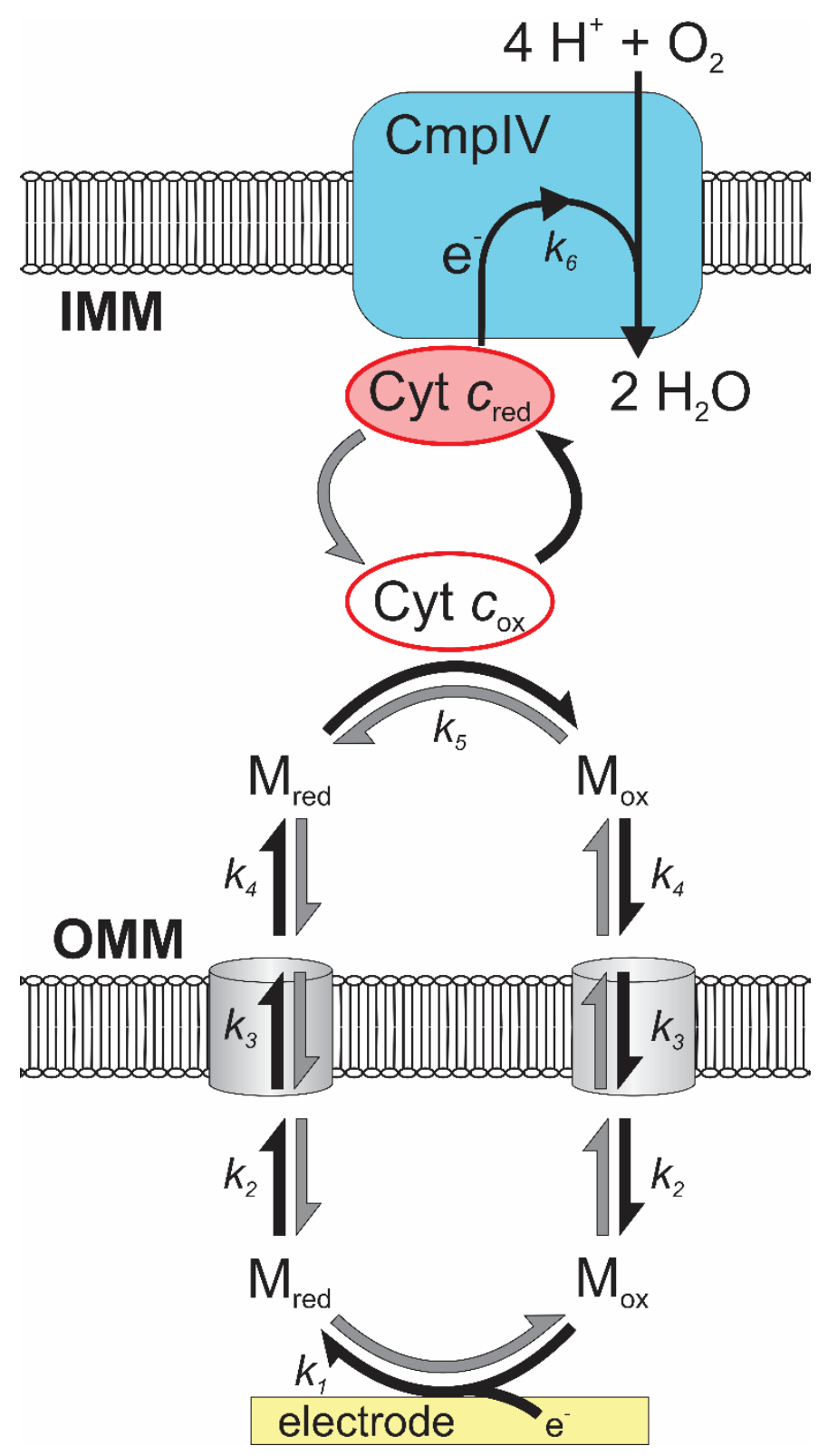

Figure S5: Mass transfer schematics of mediated mitochondrial electrochemistry. An external mediator is utilized to facilitate electron transfer across the semi-permeable outer mitochondrial membrane (OMM), which precludes direct electron transfer between $\mathrm{COX}$ or endogenous $\mathrm{CytC}$ and the electrode. In this example, oxidized mediator $\left(\mathrm{M}_{\mathrm{ox}}\right)$ is reduced at the electrode, crosses OMM, and is oxidized by the endogenous CytC. Electron transfer from $\mathrm{CytC}$ to $\mathrm{COX}$ in the inner mitochondrial membrane (IMM) results in the reduction of oxygen to water. Changes in the overall catalytic turnover rate of complex IV $\left(k_{6}\right)$ can be determined amperometrically, provided that all other steps are not rate limiting, including electron mediator reduction on the electrode $\left(k_{1}\right)$, mediator diffusion in the solution and in the mitochondrial intermembrane space ( $k_{2}$ and $k_{4}$, respectively), passive mediator transport across $\operatorname{OMM}\left(k_{3}\right)$, and bimolecular reduction of $\mathrm{CytC}\left(k_{5}\right)$. 


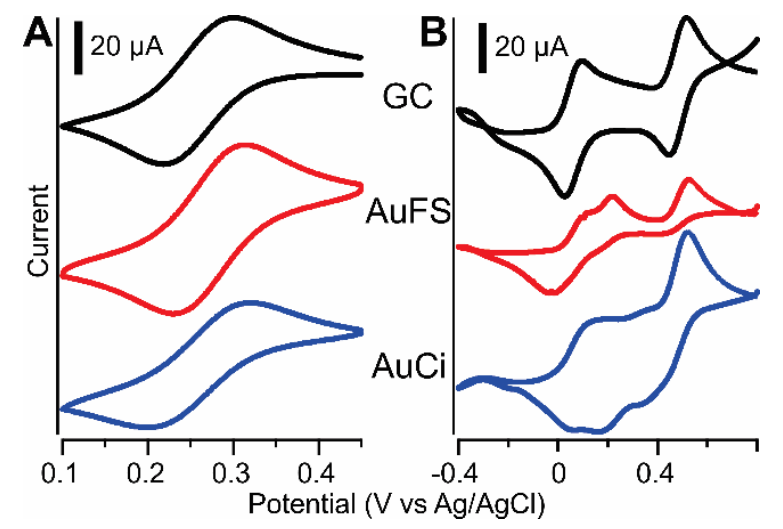

Figure S6: Electrochemical response of artificial mediators. Background-corrected CVs of $1 \mathrm{mM}$ $\mathrm{Fe}(\mathrm{CN})_{6}^{-3 / 4}$ (A) and $1 \mathrm{mM}$ TMPD (B) on glassy carbon (top, black), AuFS (middle, red), and activated $\mathrm{AuCi}$ (bottom, blue) electrodes. $\mathrm{CV}$ scan rates were $50 \mathrm{mV} \mathrm{s}^{-1}$ in the presence of $0.5 \mathrm{M} \mathrm{KCl}$. Other conditions were identical to those used in Table S1. 


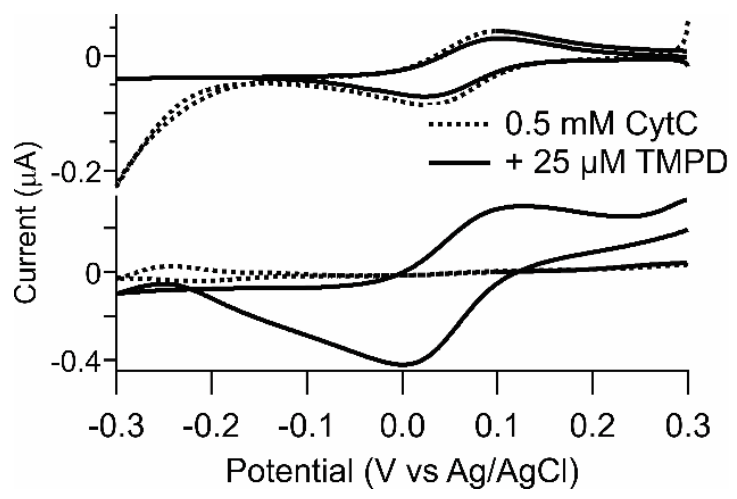

Figure S7: Electrochemical response of cytochrome $c$ on modified electrodes. CVs of $0.5 \mathrm{mM}$ CytC on AuFS (top) and activated AuCi (bottom) electrodes were measured in the absence of TMPD (dashed line) or with $25 \mu \mathrm{M}$ TMPD (solid line). Measurements were performed in $100 \mathrm{mM}$ Tris buffer, $\mathrm{pH} 7.5$, containing $0.5 \mathrm{M} \mathrm{KCl}$ in the microfluidic cell $\left(125 \mu \mathrm{m}\right.$ layer) at a scan rate of $1 \mathrm{mV} \mathrm{s}^{-1}$. CVs of blank samples without $\mathrm{CytC}$ were used for background corrections. CV of $25 \mu \mathrm{M}$ TMPD alone is indistinguishable from the background on this scale. No redox transition was observed for $\mathrm{CytC}$ on $\mathrm{AuCi}$ (bottom, dashed line) until it was rescued by the addition of TMPD (bottom). 


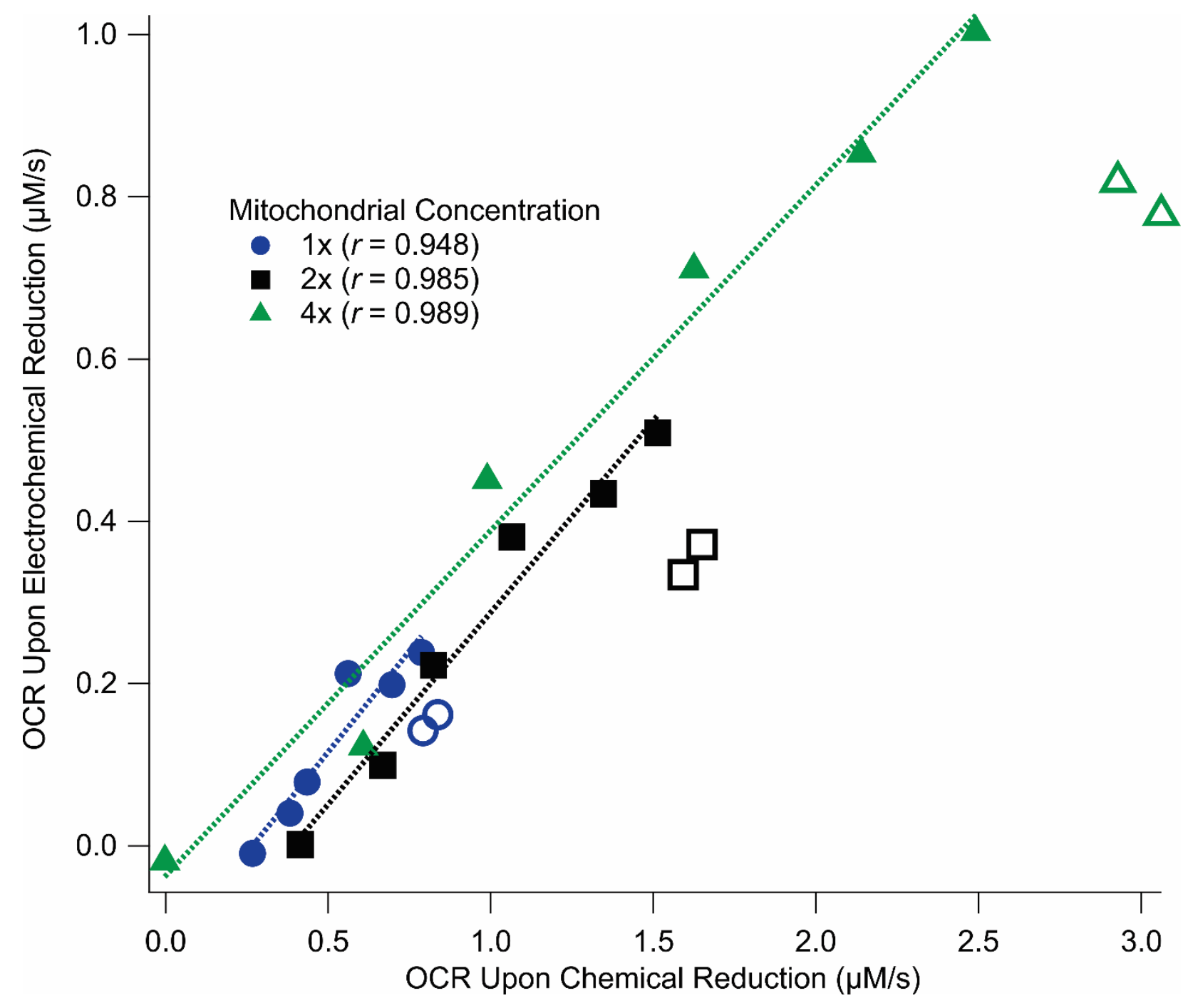

Figure S8: Correlation of mitochondrial oxygen consumption rates observed upon chemical and electrochemical reduction at various TMPD concentrations. Polarographic OCRs (horizontal axis) observed upon chemical reduction with ascorbate and microfluidic electrochemical OCRs (vertical axis) were obtained from the data shown in Figure. 5 of the main text. Only results obtained at TMPD concentrations of $1 \mathrm{mM}$ and below (solid markers) were considered in linear correlation analysis, while TMPD concentrations of $1.5 \mathrm{mM}$ and $2 \mathrm{mM}$ (open markers) were not. The Pearson correlation coefficients $r$ for each group are shown. The critical value for each of these groups is $0.707(n=6, \alpha=0.5)$. 

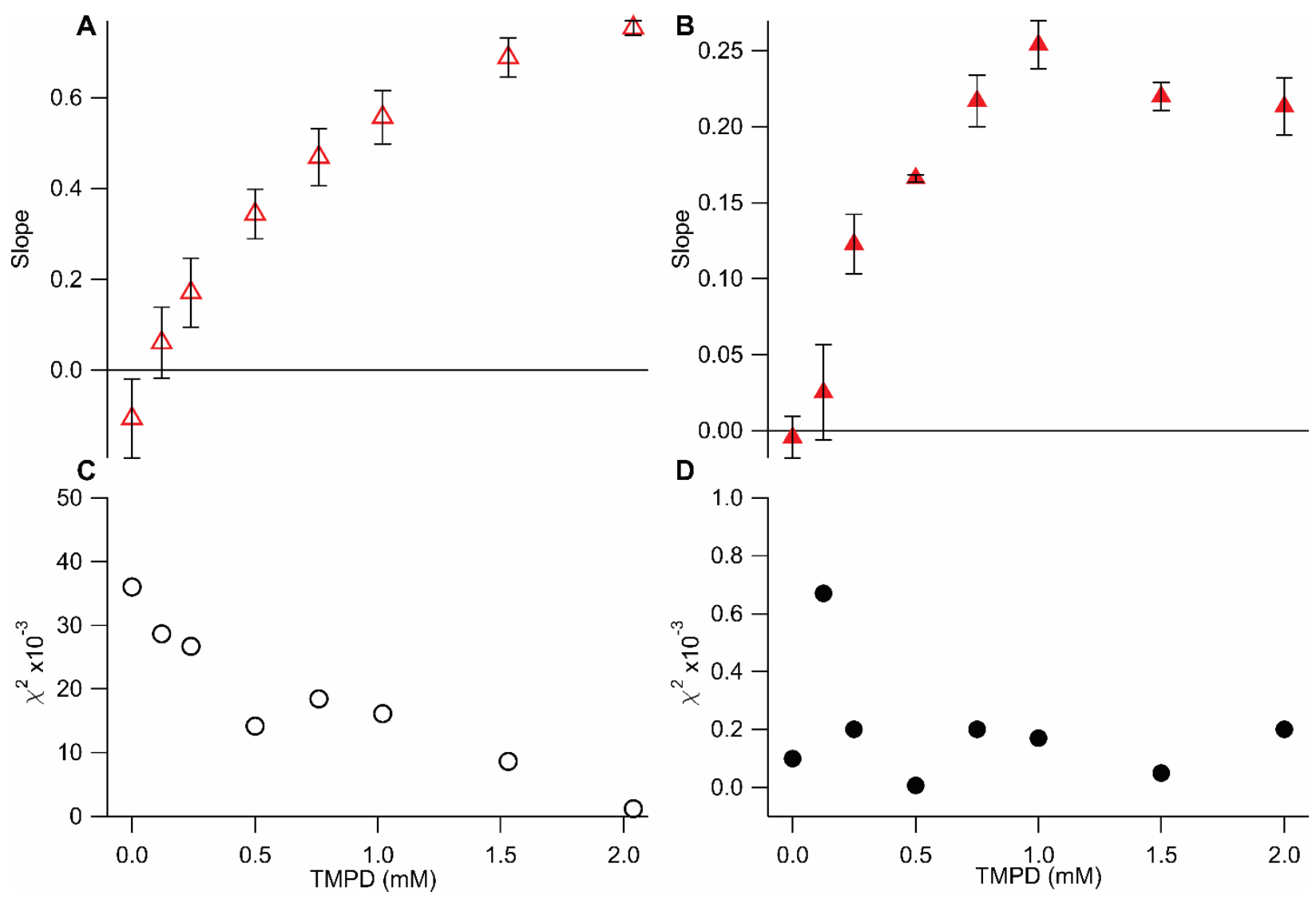

Figure S9: Linearity of OCRs with mitochondrial concentration at various TMPD concentrations.

Data presented in Figure. 5 of the main text were subjected to linear regression against relative mitochondrial density at each TMPD concentration. The values of linear slope and linear fitness $\left(\chi^{2}\right)$ are shown in the top and bottom panels, respectively. Measurements were performed either by Clark electrode upon chemical reduction with $4 \mathrm{mM} \mathrm{Na}$ ascorbate (A and $\mathrm{B}$, from Figure $5 \mathrm{~A}$ ) or in the microrespirometer upon electrochemical reduction ( $\mathrm{C}$ and $\mathrm{D}$, from Figure. 5B). Panels $\mathrm{A}$ and $\mathrm{C}$ show standard errors of the slope as reported by unconstrained linear regression in Igor Pro. 


\section{$\underline{\text { References }}$}

(1) Schauff, S.; Ciorca, M.; Laforgue, A.; Bélanger, D. Electron Transfer Processes at Aryl-Modified Glassy Carbon Electrode. Electroanalysis 2009, 21 (13), 1499-1504. https://doi.org/10.1002/elan.200804568.

(2) Konopka, S. J.; McDuffie, B. Diffusion Coefficients of Ferri- and Ferrocyanide Ions in Aqueous Media, Using Twin-Electrode Thin-Layer Electrochemistry. Anal. Chem. 1970, 42 (14), 17411746. https://doi.org/10.1021/ac50160a042.

(3) García-Miranda Ferrari, A.; Foster, C. W.; Kelly, P. J.; Brownson, D. A. C.; Banks, C. E. Determination of the Electrochemical Area of Screen-Printed Electrochemical Sensing Platforms. Biosensors 2018, 8 (2), 53. https://doi.org/10.3390/bios8020053.

(4) Kuznetsov, A. V; Gnaiger, E. Oxygraph Assay of Cytochrome c Oxidase Activity : Chemical Background Correction. Mitochondrial Physiol. Netw. 2003, 6, 1-4. 